\title{
Estudo da Expressão da Proteína Caderina-E Correlacionada com o Grau de Diferenciação Celular e o Estadiamento TNM do Adenocarcinoma Colorretal
}

\author{
Relationship Study Between the Cadherin-E Protein Cell \\ Diferentiation and TNM Staging
}

\author{
MARCOS VINICIUS ARAUJO DENADAI ${ }^{1}$;ARMANDO GERALDO FRANCHINI MELANI; \\ CARLOSAUGUSTO VÉO ${ }^{3}$; SANDRAREGINAMORINIDASILVA ${ }^{4}$
}

\begin{abstract}
${ }^{1}$ Médico do Departamento de Cirurgia Oncológica da Fundação Pio XII-Hospital do Câncer - Barretos - SP. FUNDAÇÃO PIO XII-Hospital de Câncer de Barretos: R. ANTENOR DUARTE VILLELA, 1331,BAIRRO PAULO PRATABARRETOS - SP- 14784-400 - (17)33216600; ${ }^{2}$ Médico do Departamento de Cirurgia Oncológica da Fundação Pio XII - Hospital do Câncer - Barretos- SP. FUNDAÇÃO PIO XII - Hospital de Câncer de Barretos; ${ }^{3}$ Médico do Departamento de Cirurgia Oncológica da Fundação Pio XII - Hospital do Câncer - Barretos - SP. FUNDAÇÃO PIO XII - Hospital de Câncer de Barretos; ${ }^{4}$ Médica do Departamento de Patologia da Fundação Pio XII - Hospital do Câncer - Barretos-SP.
\end{abstract}

DENADAI MVA; MELANI AGF; VÉO CA; SILVA SRM. Estudo da Expressão da Proteína Caderina-E Correlacionada com o Grau de Diferenciação Celular e o Estadiamento TNM do Adenocarcinoma Colorretal Rev bras Coloproct, 2006;26(3): 310-315.

RESUMO: Objetivo: Avaliar a relação de uma proteína que participa do mecanismo de adesão celular com o grau de diferenciação celular e o estadiamento TNM I e IV no CCR. Métodos: Foram estudados 100 pacientes (54 homens e 46 mulheres) tratados por CCR, estádio I - 44 pacientes, estádio IV - 56 pacientes. Os cortes histológicos do tecido tumoral foram examinados por técnica de imunohistoquímica em relação à expressão da proteína caderina-E. Os cortes histológicos foram classificados como positivos ou negativos pelo método semiquantitativo. Resultados: Para o TNM, expressão da caderina-E estádio I: positiva em 72,7 \% e negativa em 35,7\% ; estádio IV: positiva em 64,3\% e negativa em 35,7\%. Em relação ao grau de diferenciação celular, expressão da caderina-E; G I: positiva em $70 \%$ e negativa em 30\%; G II: positiva em $68.4 \%$ e 31,6\% negativa; G III: $63.6 \%$ positiva e 36,4 \% negativa.. Não houve diferença significativa entre os grupos. Conclusão: Os resultados dessa pesquisa permitem concluir que não há relação da expressão da proteína caderina-E com o estadiamento TNM (I e IV) e o grau de diferenciação celular no CCR.

Descritores: Caderinas; neoplasias do cólon; cólon; reto; moléculas de adesão celular.

\section{1 - INTRODUÇÃO}

O carcinoma colorretal (CCR) representa um problema de saúde pública mundial. O número de casos novos de CCR estimados para o Brasil em 2006 é de 11.390 casos em homens e de 13.970 em mulheres. (1)

Pacientes portadores de CCR em estádios iniciais apresentam bom prognóstico, porém, nos está- dios avançados da doença há uma redução da sobrevida (20\% em 5 anos), ${ }^{(2)}$ Entretanto, alguns pacientes com doença localizada morrem por recidiva tumoral, mesmo recebendo o tratamento adequado. ${ }^{(3)}$

Diante desses fatos, trabalhos na literatura foram elaborados numa tentativa de traçar um perfil prognóstico para o CCR, levando em conta não só as informações do exame anatomo-patológico e de estadiamento, mas também o conhecimento das es-

Trabalho realizado na FUNDAÇÃO PIO XII - Hospital de Câncer de Barretos- SP. Departamento de Cirurgia Oncológica - Barretos - SP - Brasil. 
truturas que participam das funções básicas da célula, como o processo de adesão celular e a capacidade de angiogênese, envolvidos também na carcinogênese. ${ }^{(4)(5)}$

A proteína caderina-E desempenha um importante papel no mecanismo de adesão celular, mediando a interação célula-célula e célula-matriz extracelular, mantendo a integridade do tecido epitelial. Pertence a uma família de glicoproteínas transmembrana, presente sobre a superfície das células, cálcio dependente. ${ }^{(6)}$

Evidências indicam que a perda da função da caderina-E está relacionada com o processo de diferenciação e metástases no carcinoma de mama e de esôfago. ${ }^{(7)(8)}$

A perda da expressão da caderina-E e das cateninas foi associada com invasão tumoral e metástases, sugerindo um potencial fator prognóstico no CCR, em trabalhos de Ghadimi et al (1999) (9) e Gofuku et al (1999). ${ }^{(10)}$

Por outro lado, Kitadai et al. (1996) ${ }^{(11)}$ não evidenciaram relação estatística da perda da expressão da caderina-E relacionada com metástases hepáticas no CCR, e Leme et al. (2005) ${ }^{(12)}$ não relacionaram a expressão da proteína caderina-E, com o estadiamento clínico e o prognóstico.

A diversidade de resultados - até controversos - em literatura específica motivou esta investigação no sentido de procurar estabelecer a relação entre a expressão da proteína caderina-E, considerada como participante do processo de adesão celular, com variáveis histopatológicas no CCR localizado (estádio I) e avançado (estádio IV).

\section{2 - OBJETIVO DA PESQUISA}

Verificar a associação entre a expressão da caderina-E com o grau de diferenciação celular e com o estadiamento TNM (estádio I e IV) do adenocarcinoma colorretal.

\section{3 - MATERIAL E MÉTODOS}

Estudo de natureza retrospectiva, desenvolvido nos Departamentos de Cirurgia Oncológica e Anatomia Patológica do Hospital do Câncer de Barretos Fundação Pio XII - Barretos -SP. As amostras biológicas foram coletadas do arquivo do Laboratório de Patologia da Fundação Pio XII e os dados clínicos foram coletados no SAME (Serviço de Arquivos Médico e Estatística).

\section{1 - Amostra}

A amostra foi constituída de 100 pacientes portadores de CCR tratados no Hospital de Câncer de Barretos - Fundação Pio XII - no período de 1993 a 2004, divididos em dois grupos de estadiamento, segundo as normas do TNM ${ }^{(13)}: 44$ pacientes estádio I (pT1 ou pT2 N0 M0) e 56 pacientes estádio IV (qualquer $\mathrm{T}$, qualquer $\mathrm{N}, \mathrm{M} 1$ ).

\section{2 - Critérios de inclusão}

Foram incluídos pacientes admitidos no hospital para tratamento, pertencendo apenas aos estádios I e IV, classificados de acordo com o sistema T $\mathrm{NM}$, possuindo prontuário médico e blocos de parafina que representam o tumor primário ou a lesão metastática, arquivados no Departamento de Patologia.

Para definir o diagnóstico e o estadiamento, foram utilizados: biópsia do tumor colorretal, exames de imagem (RX tórax, TC abdominal, US abdominal), biopsia da lesão metastática, achado intra - operatório e laudo do exame anátomo - patológico. No grupo considerado como estádio I, 6 pacientes receberam tratamento pré-operatório com radioterapia e quimioterapia, e 2 pacientes receberam apenas radioterapia neo-adjuvante, num total de 8 (18\%) pacientes em 44 casos.

\section{3 - Critérios de exclusão}

Foram considerados critérios de exclusão: indisponibilidade de dados clínicos e/ou anátomo-patológicos, estadiamentos TNM II e III, pacientes portadores de polipose familiar, outros tipos histológicos diferentes do adenocarcinoma e tumores metacrônicos.

\section{4 - Características da amostra}

Em relação ao gênero dos pacientes, ficaram distribuídos: 18 (41\%) - feminino e $26(59 \%)$ masculino no grupo estádio I e $28(50 \%)$ - feminino e $28(50 \%)$ - masculino no estádio IV. O tipo histológico predominante foi o adenocarcinoma clássico tubular.

Quanto ao grau de diferenciação celular, para o estádio I: 9 (20,4\%) - grau I; 33 (75\%) - grau II e $2(4.5 \%)$ dos tumores grau III. Nos pacientes do estádio IV, $1(1,8 \%)$ tumor foi classificado como grau I; 46 $(82,1 \%)$ eram grau II e $9(16 \%)$ tumores grau III.

Uma síntese dos resultados das variáveis está disposta no quadro 1 . 
QUADRO 1 - Distribuição dos pacientes quanto ao gênero, idade média e grau de diferenciação celular, segundo o estadiamento I e IV.

\begin{tabular}{|c|c|c|c|c|c|}
\hline \multicolumn{2}{|l|}{ ESTADIAMENTO } & \multicolumn{2}{|c|}{ TNM I / n= 44 } & \multicolumn{2}{|c|}{ TNM IV / n= 56 } \\
\hline Gênero & Masculino & 26 & $(59 \%)$ & 28 & $(50 \%)$ \\
\hline & Feminino & 18 & $(41 \%)$ & 28 & $(50 \%)$ \\
\hline Idade média (anos) & & 61 & & 55 & \\
\hline \multirow[t]{3}{*}{ Grau de diferenciação celular } & Grau I & 9 & $(20,4 \%)$ & 1 & $(1,8 \%)$ \\
\hline & Grau II & 33 & $(75 \%)$ & 46 & $(82,1 \%)$ \\
\hline & Grau III & 2 & $(4.5 \%)$ & 9 & $(16 \%)$ \\
\hline
\end{tabular}

\section{5 - Técnica Imunohistoquímica}

Para avaliação da expressão da proteína caderinaE pelo método de imunohistoquímica, foram usados anticorpos primários monoclonais: E-cadherin Novocastra NCL E-cad, clone 36 B 5, diluição de 1/ 100.

Os blocos de parafina foram recortados em micrótomo rotativo, obtendo-se cortes histológicos de 2 a 3 micra de espessura e depositados em lâminas previamente tratadas com silano (3 -aminopropyl triethoxilane, SIGMA A-3648, USA) com a reação utilizando o complexo Avidina-biotina-perxidase (ABC) / Estreptavidina-biotina-peroxidase (StreptABC).

\section{método imunohistoquímico \\ 3.6 - Avaliação dos resultados obtidos pelo}

A avaliação da expressão da proteína foi realizada por leitura das lâminas histológicas em microscópio óptico (aumento de $40 \mathrm{x}$ ), por 3 médicos patologistas que desconheciam o estadiamento do material.

O critério adotado foi o seguinte: achados considerados positivos ou normais quando se detectou a presença da proteína caderina-E na membrana celular em 50\% ou mais das células tumorais e negativos quando se verificou a ausência da proteína; localização no citoplasma; menos de $50 \%$ de células com expressão da proteína caderina-E. Os resultados foram obtidos em consenso entre os patologistas, aplicando-se o método semiquantitativo.

As figuras 1 e 2 a seguir representam a expressão desta proteína pelo método imunohistoquímico.

\section{7 - Análise estatística}

Os resultados foram submetidos a tratamento estatístico com a finalidade de determinar se existe correlação entre a expressão da proteína caderina-E com o estadiamento e com o grau de diferenciação tumoral no CCR.
O teste de qui-quadrado de Pearson foi empregado para a comparação dos dados coletados com os resultados obtidos da expressão da proteína caderina-E, adotando como nível de significância $\mathrm{p}=$ 0,05 .

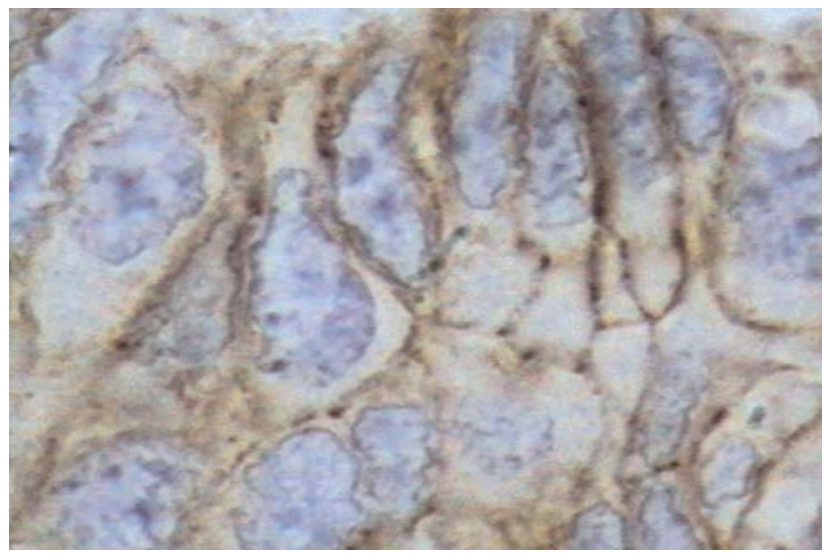

Figura 1 - Fotomicrografia do CCR com expressão da proteína caderina-E classificada como positiva na membrana celular. (aumento 100x).

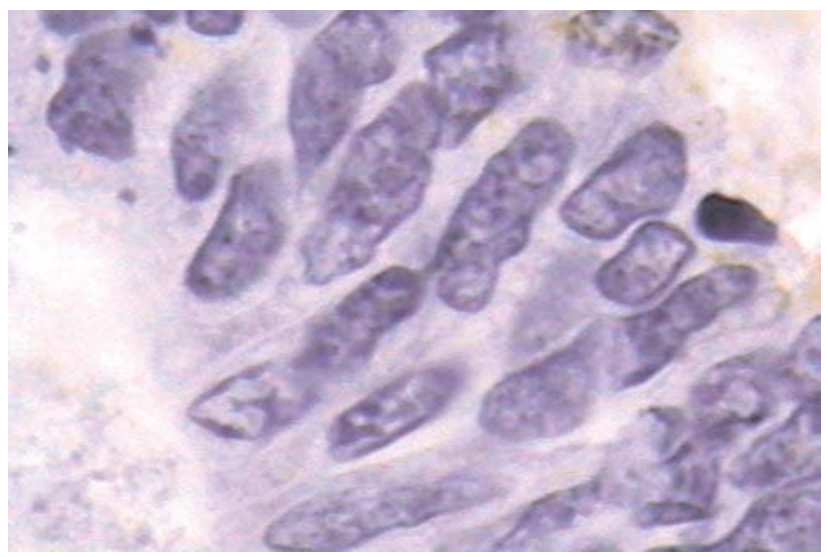

Figura 2 - Fotomicrografia do CCR com expressão da proteína caderina-E classificada como negativa na membrana celular. (aumento 100x). 


\section{4 - RESULTADOS}

\section{1 - Expressão da proteína caderina-E no estadiamento TNM}

Em relação à caderina-E para o estadiamento I: expressão positiva em 32/44 (72,7 \%) pacientes e expressão negativa em 12/44 (27,2\%) pacientes. Para o estádio IV: expressão positiva em 36/56 $(64,2 \%)$ pacientes e negativa em 20/56 (35,7\%). Não houve diferença significativa entre os grupos $(\mathrm{p}=0,37)$.

4.2 - Expressão da proteína caderina-E e grau de diferenciação celular

Para os tumores G I, expressão positiva em 7/ $10(70 \%)$ casos e negativa em $3 / 10(30 \%)$ casos. Nos tumores G II, os achados positivos totalizaram 54/79 $(68.3 \%)$ casos e $25 / 79$ (31,6\%) negativos; e nos tumores G III, 7/11 (63.6\%) casos com expressão positiva e 4/11 (36,3\%) com expressão negativa. Não houve diferença significativa observada entre os grupos $(\mathrm{p}=$ $0,94)$.

Os resultados encontrados das variáveis estudadas nos pacientes selecionados para esse trabalho estão dispostos no quadro 2.

\section{5 - DISCUSSÃO} caderina-E

\section{1 - Avaliação da expressão da proteína}

A identificação da caderina-E, pelo método imunohistoquímico, foi realizada pela detecção da coloração marrom localizada na membrana celular.

Na literatura há variação dos critérios que determinam a positividade da expressão da caderinaE. Alguns autores consideram positividade da expressão da caderina-E quando $90 \%$ das células tumorais encontravam-se coradas pelo método, enquanto que, para outros, o critério de positividade situa-se entre 25 e $50 \%$ das células coradas. ${ }^{(14)(15)}$

O resultado encontrado foi de $32 \%$ de expressão negativa para a proteína caderina-E e $68 \%$ de expressão normal, no total dos 100 pacientes estudados, estando esse valor dentro dos parâmetros detectados pelos trabalhos da literatura ${ }^{(10)(16)}$ e com diferença estatística significativa $(\mathrm{p}<0,001)$.

Vale ressaltar que a expressão da proteína foi considerada normal ou positiva em todos os pacientes (8) submetidos a tratamento prévio com radioterapia e quimioterapia.

\section{2 - Expressão da proteína caderina-E com o estadiamento TNM}

Na literatura existem estudos que identificam correlação entre a expressão da caderina-E com o estadiamento TNM, como o de Mohri et al, 1997; (17) Ikeguchi et al, $2000^{(18)}$; e o trabalho de Kaihara et al, $2003^{(19)}$, que relacionaram a perda da expressão dessa proteína com os tumores em estádios avançados.

Delektorskaya et al, 2005, evidenciaram uma importante redução ou total ausência da expressão da caderina-E em tumores colorretais com metástases hepáticas (estádio IV) quando comparada a pacientes sem metástases. (20)

Outros trabalhos revelam resultados discordantes, não encontrando relação da expressão da caderina-E com o estadiamento, como apresentado por Karatzas et al, $1999^{(21)}$; Hugh et al, $1999^{(4)}$; Jesus et al,2005 (22) ; Leme et al, $2005^{(12)}$.

Neste estudo, não se identificou correlação entre a expressão da proteína caderina-E com o estadiamento TNM, ou seja, a perda da expressão não se relacionou com o estádio mais avançado da doença.

QUADRO 2 - Resumo dos resultados verificados na avaliação da relação das proteínas caderina-E e DCC com o estadiamento TNM e o grau de diferenciação celular.

\begin{tabular}{lccccc}
\hline PROTEÍNA & \multicolumn{2}{c}{ ESTADIAMENTO } & \multicolumn{2}{c}{ GRAU DE DIFERENCIAÇÃO } \\
\cline { 2 - 6 } & TNM I & TNM IV & Grau I & Grau II & Grau III \\
\hline caderina-E positiva & $32 / 44$ & $36 / 56$ & $7 / 10$ & $54 / 79$ & $7 / 11$ \\
& $72,7 \%$ & $64,2 \%$ & $70 \%$ & $68,3 \%$ & $63,6 \%$ \\
caderina-E negativa & $12 / 44$ & $20 / 56$ & $3 / 10$ & $25 / 79$ & $4 / 11$ \\
& $27,2 \%$ & $35,7 \%$ & $30 \%$ & $31,6 \%$ & $36,3 \%$ \\
\hline
\end{tabular}


Existem diferenças metodológicas entre os trabalhos citados, tanto na leitura dos resultados obtidos pela imunohistoquímica, quanto na comparação com os estadiamentos do sistema TNM, não havendo, portanto, uma padronização adotada. Este fato pode interferir na análise quando se comparam os resultados da literatura.

Os trabalhos de Leme et al, $2005^{(12)}$ e Jesus et

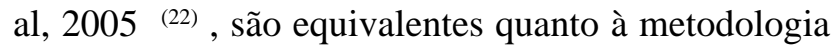
em relação ao TNM, comparando todos os estádios da doença com a expressão da proteína caderina-E, não encontrando relação estatística, reforçando a hipótese de que essa proteína não participa do processo de progressão tumoral.

5.5 - Expressão da proteína caderina-E com o grau de diferenciação celular

Em relação ao grau de diferenciação celular, neste estudo, houve um predomínio de tumores grau II (moderadamente diferenciados): $75 \%$ nos tumores estádio I e $83,9 \%$ nos casos do estádio IV, fato também observado em outros trabalhos da literatura. ${ }^{(15)}$

Guzinska-Ustymowicz et al, 2004, estudando a expressão da caderina-E em 34 pacientes com CCR, classificados pelo exame anátomo patológico como pT1, por imuno-histoquímica, evidenciaram forte relação com a perda da expressão e o grau histológico. ${ }^{(23)}$
Outros estudos não mostraram relação da caderina-E com a diferenciação celular, conforme citados a seguir: Ilyas et al, $1997^{(15)}$, analisando 68 casos de tumores colorretais, não encontraram relação significativa com o grau de diferenciação; Kaihara et al, $2003^{(19)}$ e Jesus et al, $2005^{(22)}$, analisando 117 pacientes, não identificaram correlação entre a proteína caderina-E com o grau de diferenciação celular.

No presente estudo, não foi encontrada correlação entre a expressão da caderina-E com o grau de diferenciação celular. Esse fato pode estar relacionado com o grande número de tumores grau II encontrados e ao reduzido número de tumores grau III nessa amostra, podendo acarretar alterações na análise estatística.

\section{6 - CONCLUSÕES}

Os resultados desta pesquisa sugerem a necessidade de estudos adicionais, utilizando métodos validados, padronizados e reprodutíveis, dos quais se possa lançar mão, com mais confiança; do uso de outros recursos além do estadiamento clínico-patológico para uma abordagem do adenocarcinoma colorretal.

Não há relação entre a expressão da caderinaE com o estadiamento TNM e o grau de diferenciação celular no CCR.

ABSTRACT: Objective: To evaluate the relationship of a protein that take part in the same mechanism of cell adhesion with the cell differentiation degree and TNM staging I and IV in CRA. Methods: One-hundred patients (54 men and 46 women), who have received treatment for CRA, stage I - 44 patients and stage IV - 56 patients, have been studied. Histological cuts of tumor tissue were examined by the immunohistochemical technique as to the expression of E-cadherin proteins. Such histological cuts were classified as positive or negative through the semi-quantitative method. Results: For TNM, the E-cadherin expression for stage I: positive in $\mathbf{7 2 . 7 \%}$ and negative in $35.7 \%$; stage IV: positive in $64.3 \%$ and negative in $35.7 \%$. Regarding the cell differentiation degree, the expression of E-cadherin, GI: positive in $70 \%$ and negative in $30 \%$; GII: positive in $68.4 \%$ and negative in $31.6 \%$; GIII: positive in $63.6 \%$ and negative in $36.4 \%$. There was no significant difference among the groups. Conclusion: The results of this research come to the conclusion that there is no relationship between the expression of E-cadherin protein with TNM staging (I and IV) and cell differentiation degree in CRA.

Key words: Cadherin; Neoplasm; Colon; Rectum; Cell cadherin.

\section{REFERÊNCIAS}

1. Instituto Nacional do Câncer. Estimativas de câncer no Brasil 2006 [monografia na internet]. Rio de Janeiro: INCA;2006. [acesso em 2006 fev 20] Disponível em: http:// www.inca.gov.br/estimativa/2006/

2. Newlan RC, Dent OF, Chapuis PH, Bokey L. Survival after curative resection of lymph node negative colorectal carcino- ma. A prospective study of 910 patients. Cancer 1995; 76: 564-71.

3. Shibata, D. The DCC protein and prognosis in colorectal cancer. N Engl J Med 1996; 335: 1727-32.

4. Hugh TJ, Dillon SA, O`Dowd G, Getty B, Pignatelli M, Poston GJ. Bet- catenin expression in primary and metastatic colorectal carcinoma. Int J Cancer 1999; 82: 504-11. 
5. Kubo H, Mihi C, Kusunoki M. Evaluation of genetic mutations of tumor suppresser genes in colorectal cancer patients. Hepatogastroenterology 2004; 51: 114-7.

6. Takeichi M. The cadherins: Cell-cell adhesion molecules controlling animal morphogenesis. Development 1988; 102: 639-55.

7. Miyata M. Relationship between E-cadherin expression and lymph node metastasis in human esophageal cancer. Int J Oncol 1994; 4: 61-5.

8. Oka H, Shiozaki H, Kobayashi K, Inoue M, Tahara H, Kobayashi T. Expression of E-cadherin cell adhesion molecules in human breast cancer tissues and relationship to metastasis. Cancer Res 1993; 53: 1696-701.

9. Ghadimi BM. Immunohistological analysis of E-cadherin, á, â catenin expression in colorectal cancer: Implications for cell adhesion and signaling. Eur J Cancer 1999; 35: 60-5.

10. Gofuku J, Shiozak H, Tsujinaka T, Inoue M, Tamura S, Doki Y. Expression of E-cadherin and alfa-catenin in patients whith colorectal carcinoma.Am J Clin Pathol 1999; 111: 29-37.

11. Kitadai Y, Ellis L M, Tucker S L, Greene G F , Bucana C D, Cleary K R. Multiparametric in situ mRNA hybridization analysis to predict disease recurrence in patients with colon carcinoma. Am J Pathol 1996; 149: 1541-51.

12. Leme MBP, Waitzberg AFL, Artigiani R, Matos D. A relação da caderina-E com o prognóstico do adenocarcinoma colorretal. Rev Col Bras Cir 2005; 32(4): 201-4.

13. AMERICAN JOINT COMMITTEE ON CANCER. Colon and rectum. Philadelphia: lippincott-Raven, 2002.

14. Karatzas G. E-cadherin expression correlates white tumor differentiation in colorectal cancer. Hepatogastroenterology 1999; 46: 232-5

15. Ilyas M, Tomlinson IP, Hanby A, Talbot IC, Bodmer WF. Allele loss, replication errors and loss of expression of Ecadherin in colorectal cancers. Gut 1997; 40: 654-9

16. Garinis GA. Hypermethylation associated transcriptional silencing of E- cadherin in primary sporadic colorectal carcinomas. J Pathol 2002; 198: 442-9.
17. Mohri Y. Prognostic significance of E- cadherin expression in human colorectal cancer tissue. Surg Today 1997; 27: 606-12.

18. Ikeguchi M, Taniguchi M, Makino M, Kaibara N. Reduced Ecadherin expression and enlargement of cancer nuclei strongly correlate with hematogenic metastasis in colorectal adenocarcinoma. Scand J Gastroenterol 2000; 35: 839-46.

19. Kaihara T. Dedifferentiation and decreased expression of adhesion molecules, E- cadherin and ZO-1, in colorectal cancer are closely related to liver metastasis. J Exp Clin Cancer Res 2003; 22(1): 117-23.

20. Delektorskaya VV, Perevoshchikov AG, Golovkov DA, Kushlinskii NE. Expression of E- cadherin, beta- catenin, and CD- 44v6 cell adhesion molecules in primary tumors and metastases of colorectal adenocarcinoma. Bull Exp Biol Med 2005; 139(6): 706-10.

21. Karatzas G. E-cadherin expression correlates white tumor differentiation in colorectal cancer. Hepatogastroenterology 1999; 46: 232-5.

22. Jesus EC, Matos D, Artigiani R, Waitzberg AF, Goldenberg A, Saad SS. Assessment of staging, prognosis and mortality of colorectal cancer by tumor markers: receptor erbB-2 and cadherins. Acta Cir Bras 2005; 20(6): 422-7.

23. Guzinska-Ustymowicz K, Chetnik A, Kemona A. Effects of changes at the site of E-cadherin expression as an indicator of colon cancer aggressiveness. Rocz Akad Med Bialymst 2004; 49: 70-2.

\section{Endereço para correspondência:}

MARCOS VINICIUS ARAÚJO DENADAI

Avenida Brigadeiro Eduardo Gomes, 178

Bairro Aeroporto

14.783-131 - Barretos (SP)

(17) $3325-3210$

E-mail:mfdenadai@uol.com.br 